\title{
Double-Blind Randomized Control Trial to Evaluate Efficacy of Tramadol for Pain Relief during the Electromyography of Lumbar Region and Pelvic Extremities
}

\author{
Ingrid Morales-Sanchez ${ }^{1,2 *}$, Sai N. Alla², Arturo Llanes-Ojeda ${ }^{3}$, Julio C. López-Valdés ${ }^{2,3}$ and \\ Fiacro Jimenez-Ponce ${ }^{2,4,5}$
}

${ }^{1}$ Department of Rehabilitation, Hospital General De México "Dr. Eduardo Liceaga," Mexico City; ${ }^{2}$ Department of Medical Research, Cognitive Science A.C., Mexico City; ${ }^{3}$ Department of Research, Autonomous University of Tamaulipas, School of Medicine "Dr. Alberto Romo Caballero" Tampico, Tamaulipas; ${ }^{4}$ Service of Neurology and Neurosurgery, Hospital General de México "Dr. Eduardo Liceaga," Mexico City; ${ }^{5}$ Department of Research, Hospital Ángeles de Pedregal, Mexico City. Mexico

\begin{abstract}
Introduction: An electromyography (EMG) is the registration of bioelectrical activity generated by the muscle through a needle electrode to provide information on the degree or type of neurological injury. Pain during the procedure is a common obstacle. Aim: This study aims to measure the effect of tramadol against a placebo on the reduction of somatic pain during the EMG intervention. Patients and Methods: A double-blind, randomized control trial was conducted in the "Hospital General de México,"from October 2012 to April 2013; 84 adult patients, aged 18-65 years, of either gender, who were diagnosed with chronic lumbar radiculopathy and scheduled for an elective EMG were consecutively enrolled for this study. Results: A total of 57 patients participated in the study. The gender ratio was 1:1; the population age was $47.16 \pm 13.31$ years. In decreasing order, the most affected nerves were L5 (40.4\%), L4 (31.6\%), S1 (7\%), and non-specific or multilevel radiculopathy in 12 patients $(21.1 \%)$. The initial pain threshold $\left(5.58 \pm 1.92 \mathrm{~kg} / \mathrm{cm}^{2}\right)$ and the final pain threshold $\left(5.33 \pm 1.79 \mathrm{~kg} / \mathrm{cm}^{2}\right)$ were determined. Conclusions: The administration of tramadol $(30 \mathrm{mg})$ before undergoing an EMG of the lower extremities was not effective for decreasing pain intensity during this procedure.
\end{abstract}

Key words: Electromyography. Tramadol. Pain. Pain threshold. Visual analog scale.

\section{Introduction}

An electromyography (EMG) is a neurophysiological study considered as an extension of a neurological examination for the evaluation of neuromuscular diseases ${ }^{1}$. EMG is the registration of bioelectrical activity generated by the muscle through a needle electrode, to identify subclinical alterations and provide objective information about the degree or type of neurological injury ${ }^{2-4}$. Unfortunately, the electrodiagnosis approach can be an uncomfortable experience for the patient. The pain sensation perceived during this procedure can make diagnosis difficult because it can distort the results ${ }^{5-9}$.

Pain during EMG procedure is a common obstacle, as a result of limited cooperation from the patient. The exploration needle, for some patients, is an extremely painful experience ${ }^{6,10}$. This creates psychological problems such as anxiety, fear, and dissatisfaction. For this reason, the use of analgesics was recommended during EMG procedure but has not been established as a routine practice for all patients ${ }^{3}$. Nonsteroidal

\section{Correspondence:}

Ingrid Morales Sánchez

E-mail: ismoraless@gmail.com
Available online: 18-01-2019

Date of acceptance: 26-09-2018

DOI: 10.24875/RMU. 18000007
Medicina Universitaria. 2018;20(4):145-151 www.medicinauniversitaria.org CC BY-NC-ND license (http://creativecommons.org/licenses/by-nc-nd/4.0/). 


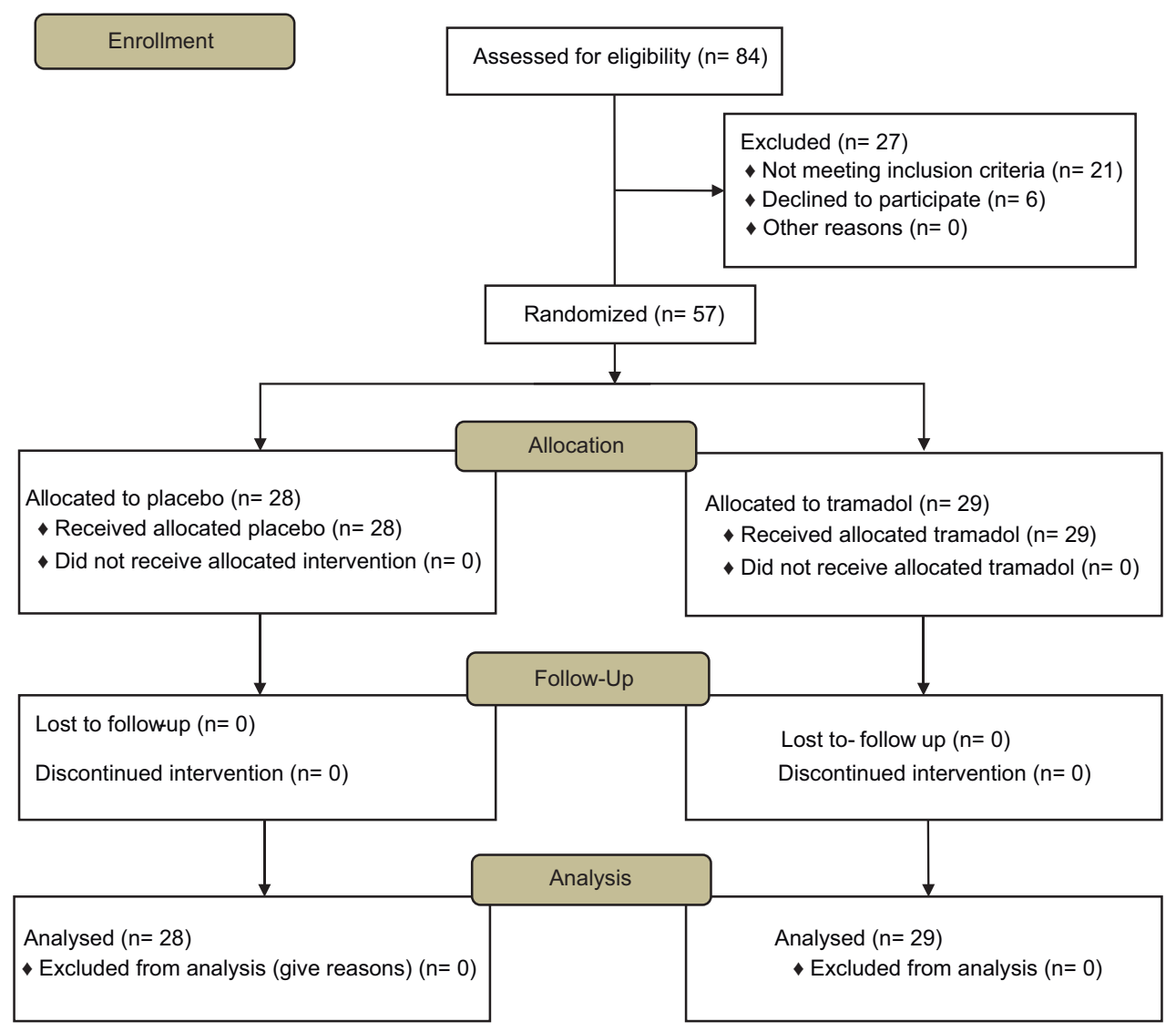

Figure 1. Distribution of patients according to the CONSORT guidelines. Modified from: Schulz KF, Altman DG, Moher D. for the CONSORT Group18 $\left({ }^{*} n=95 \% \beta=0.87\right)$. Patients with no informed consent signed $(n=6,9.3 \%)$, no complete selection criteria $(n=21,32.81 \%)$; patients with acute analgesic drugs $(n=9,14.06 \%)$, chronic pain diagnosis $(n=8$, $12.5 \%)$, older than 65 years $(n=2,3.1 \%)$, and analphabetic $(n=2,3.1 \%)$ were excluded from the study.

anti-inflammatory drugs (NSAIDs) have an analgesic effect on acute as well as mild-to-moderate chronic pain, but none of these medications deals with the problem perfectly, hence, the search for a new drug.

Some studies have shown that tramadol is an effective pain-relieving therapy for somatic pain, with few adverse effects at low doses $(<50 \mathrm{mg} / \text { day })^{11-16}$.

This double-blind, randomized study was planned to determine the efficacy, tolerability, and acceptability of $30 \mathrm{mg}$ of an oral solution containing tramadol for reducing pain intensity in patients undergoing an EMG procedure of the pelvic extremities.

\section{Patients and Methods}

A double-blind, randomized control study was conducted at the Rehabilitation Department of the "Hospital General de México," from October 2012 to April 2013. Following written and informed consent, 84 adult patients, aged 18-65 years, of either gender, who were diagnosed with chronic lumbar radiculopathy and scheduled for an elective EMG test, were consecutively enrolled for this study. Patients with a medical history of hypersensitivity to tramadol, drug abuse, or any other neurological disease that could cause chronic pain manifestations were excluded from the study. All the study participants were aware of the nature, objectives, benefits, and risks of the project.

Patients were randomly allocated into one of two groups (tramadol vs. placebo) using computer software (Epidat V 3). Group I (control) received $30 \mathrm{ml}$ of a flavored placebo beverage; Group II (tramadol) received $30 \mathrm{mg}$ of a tramadol oral solution, equivalent to 20 drops, diluted in $30 \mathrm{ml}$ of flavored beverage. Assuming that tramadol therapy would decrease pain by $30 \%$, a power calculation with $\alpha=0.05$ and $\beta=0.80$ required enrollment of at least 28 patients in each group. The process of distribution for the patients (Fig. 1 CONSORT ${ }^{17}$ ) is observed in figure 1. 
After the random assignment of the group, all patients underwent a test to determine their pain threshold pressure using a manual algometer (Pain Diagnosis and Therapeutics ${ }^{\circledR}$ ) applied at the junction of the proximal third with the medial anterior tibial edge, and the data obtained were documented. Assessment of pain was done by a $10 \mathrm{~cm}$ visual analog scale (VAS) of $0-10$, where 0 stands for "no pain" and 10 stands for "the worst imaginable pain." After the determination of the patients' pain thresholds, the Hamilton anxiety and depression scale was applied to each patient.

An associate researcher administered the beverage (flavored vs. drug) to the patient in line $30 \mathrm{~min}$ before EMG in the lumbar region and pelvic extremities; the study was always performed by the same researcher (a rehabilitation specialist). EMG was performed with Neuromax EMG ${ }^{\circledR} 1004$ equipment using a disposable monopolar $50 \mathrm{~mm}$ electrode needle and a surface electrode disk type operating as a reference, both connected to the amplifier equipment. Three muscles were explored by myotome, inserting the electrode needle into the corresponding muscle motor point according to the anatomical guide by Perotto EMG ${ }^{18}$. After insertion of the needle, exploration of four fields in each of the quadrants of the muscle was performed both in the lumbar region and lower extremities; the above was assessed according to the guidelines established by the American Association of Electrodiagnostic Medicine in the evaluation of lumbar radiculopathy protocol.

For the study, the patients were asked to lie down comfortably in a decubitus position to assess the paravertebral muscles and posterior pelvic extremities. Later, the patient was placed in a decubitus supine position to assess the anterior, lateral, and medial muscles of the pelvic extremities.

The muscles that were studied at each level were the paraspinal lumbosacral (level L1-S1), the vastus lateralis of the quadriceps (level L2-L4), the tibialis anterior (L4, L5), the peroneus longus (level L5, S1), and the medial gastrocnemius and the gluteus maximus (level S1, S2).

After EMG, the same physician measured the patient's pain threshold. Later, in the absence of the physician who performed EMG, a research associate systematically provided instructions to the patient to qualify pain intensity globally using the VAS.

VAS is a straight line of $10 \mathrm{~cm}$ with well-defined limits; the starting point indicates no pain, and the extreme end indicates the most intense pain. VAS was taken, and the distance in millimeters from the starting point of the line to the line marked by the patient was recorded. The data were entered into the data collection sheet and then transferred to a database. Information about the presence of adverse effects was also collected by drawing up a checklist at the end of the study.

Determination of pain threshold was used as a reference for the comparison of both groups partially controlling the confounding factor, measuring pain intensity during the study, and allowing a correlation between VAS score and the threshold value of pain.

Data allocation, somatometry, and the vital signs of patients were entered in a log record that remained under safekeeping until completion of the experimental phase of the maneuver to maintain the blinding of both patient and examiner.

\section{Statistical analysis}

All measured values were presented as mean \pm standard deviation and numbers (\%). A database was used to describe the demographic characteristics and the Student's $t$-test for hypothesis testing of the means of VAS that was obtained from each group.

Given the ethical basics, it was not considered proper to continue subjecting the patients to a painful workup after assessing the aim of this study. For that reason, the study was divided into three phases (Phase 1: $n=20$, Phase 2: $n=40$, and Phase 3: $n=57$ ) to detect significant differences between groups for mean VAS score. Likewise, the interim analysis was performed by one of the researchers only to decide whether the project should continue or be concluded, without providing more details of the analysis. This allowed us to maintain blinding during all phases of the study and avoid biased treatment or care.

Demographical data (age, gender, body mass index [BMI], anxiety, and EMG diagnosis) were converted to nominal and ordinal variables, due to the need for a stratified analysis and to control potential confounders. The variables were treated by non-parametric statistics: the Mann-Whitney U-test in the case of age (age group; $Z=0.16, p=0.87$ ), BMI (classified as normal, overweight or obese; $Z=1.2, p=0.22$ ), and anxiety (anxiety levels; $Z=0.94, p=0.34$ ). For EMG diagnosis variable (dichotomized in healthy or sick), the Fisher test was used.

The SPSS 17 (SPSS Inc. Chicago, IL; USA) package was used for statistical analysis. A value of $p<0.001$ was considered statistically significant. 
Table 1. General description of the sample population

\begin{tabular}{|l|c|}
\hline Variable & Mean \pm SD \\
\hline Sex (\%) & - \\
\hline Female 29 (50.9) & - \\
\hline Male 28 (49.1) & - \\
\hline Age (years) & $47.16 \pm 13.31$ \\
\hline BMI^ & $28.62 \pm 4.27$ \\
\hline Evolution time (months) & $25.7 \pm 40.67$ \\
\hline Cardiac frequency (bpm) & $73.8 \pm 11.16$ \\
\hline Respiratory frequency (rpm) & $17.4 \pm 2.50$ \\
\hline Blood pressure (mmHg) & $113 / 72 \pm 12.8 / 7.9$ \\
\hline IPT (kg/cm²) & $5.58 \pm 1.92$ \\
\hline FPT (kg/cm²) & $5.33 \pm 1.78$ \\
\hline VAS (mm) & $41.7 \pm 24.4$ \\
\hline Study duration (min) & $18.47 \pm 3.11$ \\
\hline Hamilton scale & $11.37 \pm 5.77$ \\
\hline
\end{tabular}

BMI^: body mass index, IPT: initial pain threshold, FPT: final pain threshold, VAS: visual analog scale.

\section{Results}

The gender ratio was $1: 1$, a slight predominance was shown in females $(50.9 \%)$. The population age was $47.16 \pm 13.31$ years, and the most common diagnosis was chronic lumbago. The most affected nerves were L5 (40.4\%), L4 (31.6\%), S1 (7\%), and 12 patients suffered from non-specific or multi-level radiculopathy $(21.1 \%)$. The evolution time for the clinical condition was $28.24 \pm 35.78$ months (range: 1 month-10 years). There was a somatometric tendency to be overweight $\left(\mathrm{BMI}=28.62 \pm 4.27 \mathrm{~kg} / \mathrm{cm}^{2}\right)$.

For EMG intervention, $57.9 \%$ of patients showed no alterations during the procedure. Furthermore, the diagnoses of lumbar radiculopathy and other neurophysiological diagnoses (e.g., polyneuropathy or peripheral nerve injury) were confirmed in $29.8 \%$ and $12.3 \%$ patients, respectively. The initial pain threshold (IPT $=5.58 \pm 1.92 \mathrm{~kg} /$ $\mathrm{cm}^{2}$ ) and the final pain threshold (FPT $=5.33 \pm 1.79 \mathrm{~kg} /$ $\mathrm{cm}^{2}$ ) were determined, and the difference between both values was calculated (Table 1).

Anxiety was considered as an intrinsic patient pain perception modifier and, in consequence, was assessed by heteroapplication of the Hamilton scale for anxiety. The score obtained in both groups was $11.37 \pm 5.77$ points. In this case, the Student's $t$-test was used (Table 2).

To compare the value of IPT, which did not show a normal distribution using the Kolmogorov-Smirnov test
( $p=0.045$ ), the Mann-Whitney U-test was applied, and a statistically significant result $(Z=-2.70, p=0.01)$ was obtained.

The comparison between the means for VAS for pain in the tramadol group $(37.97 \pm 24.64 \mathrm{~mm})$ versus the placebo group $(45.64 \pm 24.02 \mathrm{~mm})$ showed no significant difference by Mann-Whitney U-test $(Z=-1.20$, $p=0.23$ ). The duration of the study in the tramadol group was $18.31 \pm 2.95 \mathrm{~min}$ against the placebo group's $18.64 \pm 3.31 \mathrm{~min}$, with no statistically significant difference $(t=-0.40, p=0.69)$ (Figs. 2 and 3 ).

\section{Discussion}

The results showed no difference between the tramadol group and the placebo group in the intensity of pain suffered by patients during and after the EMG procedure. Therefore, the proposed hypothesis regarding the clinical utility of tramadol to relieve pain in this kind of diagnostic study is rejected. The authors expected a difference of at least $20 \mathrm{~mm}$ in VAS score from one group to another. However, the difference obtained in the study was only $7.67 \mathrm{~mm}$, associated with a high standard deviation in both cases (tramadol $24.64 \mathrm{~mm}$ and placebo $24.02 \mathrm{~mm}$ ). No severe presence of adverse events was observed.

In our study, the value for VAS was higher $(41.7 \pm$ $24.43 \mathrm{~mm}$ ), then VAS scores reported by El-Salem and Shakhatreh $(25 \mathrm{~mm})^{19}$ and Smith and Tong $20(29 \mathrm{~mm})$. However, both studies only explored the extremities, the current study included muscles of the paravertebral lumbar region and pelvic extremities.

Previous reports about pain relief during an EMG have shown that different maneuvers and mechanisms, along with analgesics, do not have positive results. Therefore, another method used was lidocaine iontophoresis ${ }^{21}$ : decreased VAS scores were obtained in subjects who received iontophoresis with lidocaine, but also in those who received iontophoresis with a placebo. Therefore, they concluded that the effect of the decrease in VAS was more likely due to the iontophoresis than the drug administered. Their results were difficult to generalize due to the small sample size $(n=14)$ and EMG examinations (only the opponent's policies muscle of both hands was assessed). Concerning the obtained VAS delta $(2 \mathrm{~cm})$, much higher than that obtained in our research, this difference was observed in the intervention and control group, which is detrimental to the biological plausibility of the hypothesis. 
Table 2. Comparison of variables by treatment group

\begin{tabular}{|c|c|c|c|}
\hline \multirow[t]{2}{*}{ Variables } & Tramadol group & Placebo group & \multirow[t]{2}{*}{ ep } \\
\hline & Mean (SD) & Mean (SD) & \\
\hline Sex & Female: 12/Male: 17 & Female: 17/Male: 11 & ----- \\
\hline Age (years) & $47.10(13.78)$ & $47.21(13.06)$ & 0.97 \\
\hline BMI & $29.39(3.35)$ & $27.83(4.99)$ & 0.17 \\
\hline Evolution period (months) & $30.95(39.15)$ & $25.43(32.39)$ & 0.56 \\
\hline Cardiac frequency (bpm) & $72.83(9.73)$ & $74.96(12.5)$ & 0.47 \\
\hline Respiratory frequency (rpm) & $16.76(2.40)$ & $18.25(2.42)$ & 0.07 \\
\hline Blood pressure $(\mathrm{mmHg})$ & $113 / 72(12 / 7)$ & $113 / 72(11 / 8)$ & $0.96 / 0.81$ \\
\hline IPT $\left(\mathrm{kg} / \mathrm{cm}^{2}\right)$ & $6.2(1.9)$ & $4.95(1.75)$ & $0.01\left({ }^{\circ} \mathrm{p}\right)$ \\
\hline FPT $\left(\mathrm{kg} / \mathrm{cm}^{2}\right)$ & $5.74(1.71)$ & $4.91(1.78)$ & 0.07 \\
\hline VAS $(\mathrm{mm})$ & $37.97(24.64)$ & $45.64(24.02)$ & $0.22\left({ }^{\circ} \mathrm{p}\right)$ \\
\hline Study duration (min) & $18.31(2.95)$ & $18.64(3.31)$ & 0.69 \\
\hline Hamilton scale & $10.0(5.88)$ & $12.75(5.40)$ & 0.07 \\
\hline
\end{tabular}

\&p: value for statistical Student's t-test. ${ }^{\circ} \mathrm{p}$ : value for the statistic Mann-Whitney U-test.

IPT: initial pain threshold, FPT: final pain threshold, VAS: visual analog scale.

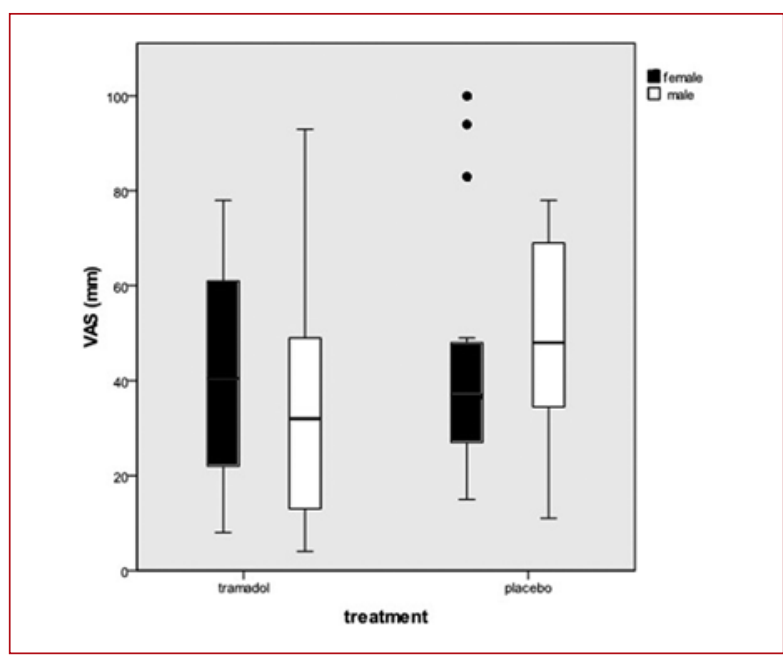

Figure 2. Comparison of visual analog scale median by gender (male vs. female) and intervention; i.e.: tramadol (Female: 12/Male: 17) versus placebo (Female: 17/Male: 11 ) by Mann-Whitney U-test $(Z=-1.20, p=0.23)$.

Unlike our results, El-Salem and Shakahtreh ${ }^{19}$ (who used ibuprofen as a painkiller for EMG) obtained statistically significant differences for VAS scale in each of the groups. However, the clinical trial they performed was self-controlled, with a small sample of healthy, young subjects with exploration of thoracic muscles

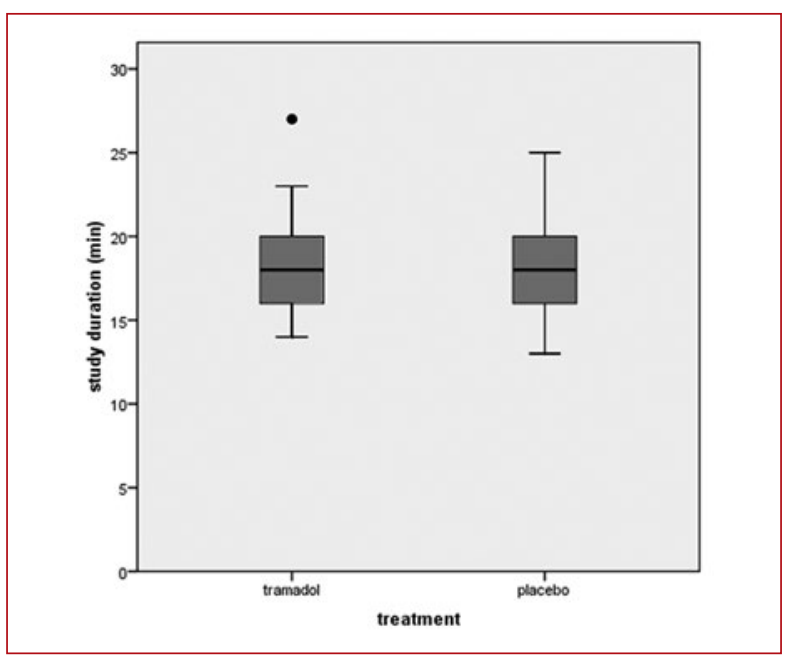

Figure 3. Comparison of study duration of EMG per intervention group; i.e., tramadol $(n=29)$ versus placebo $(n=28)$, by the Student's $t$-test $(t=-0.40, p=0.69)$.

(three muscles) and pelvic extremities (only the tibialis anterior). Being a self-controlled study, the variability of VAS decreased in comparing the same subjects, reducing the dispersion of data, which facilitates obtaining a statistically significant result with a small sample (possible error type I). Furthermore, the difference of 
the effect presented in this study was small (the difference between the means was only $8 \mathrm{~mm}$ between the ibuprofen and placebo group), quite similar to the results obtained in our study $(7 \mathrm{~mm})$. These values have no clinical significance, and thus, implementing the use of any of these drugs in daily practice would not provide a greater benefit to the patient.

With the intention of obtaining a more objective measure for the perception of pain, the pain threshold was measured before drug administration and once EMG was performed. The values obtained for each of the groups coincide with those reported in the literature 22 (average $=5.58 \mathrm{~kg} / \mathrm{cm}^{2}$ ) for the tibial region or pelvic limbs. The difference between the initial and final measurement was nominal $\left(0.25 \mathrm{~kg} / \mathrm{cm}^{2}\right)$, which is an indication of the consistency of the measure and of the fact that the tramadol mechanism of action does not alter the pain threshold, but the transmission of painful stimuli. The nociceptive stimulus for each of these assessments was almost the opposite since the electrode needle is a pointed and sharp object. The painful stimulus of the algometer was applied with pressure from a flat area against the explored body surface ${ }^{3}$, which could explain that the expected correlation between VAS and the pain threshold is not present.

Despite having made prior randomization to enroll the patients, it was observed that the IPT variable was different between groups. The indication of an IPT in the placebo group (mean $=4.95 \mathrm{~kg} / \mathrm{cm}^{2}$ ) and a difference of $1.25 \mathrm{~kg} / \mathrm{cm}^{2}$ between the two groups (meaning tramadol $6.2 \mathrm{~kg} / \mathrm{cm}^{2} ; \mathrm{p}=0.01$ ), shows a possible implication: the intervention group had some "advantage" over the placebo group and therefore, there is a risk of a type I error if the hypothesis had been accepted. However, it can be considered that this initial difference between groups does not affect the results of the outcome because it lacks biological plausibility.

Another feature of the sample is the degree of patient anxiety, measured by the Hamilton scale, which, in our case, was $52.6 \%$ for mild anxiety and $26.3 \%$ for severe anxiety. That is, only $21.1 \%$ of patients that had no anxiety before the study. Because it was considered that anxiety could be a factor that changes the perception of pain, this result was considered a confounding variable. Nevertheless, no difference between the groups or the outcome was observed. So far, the results of the research that evaluates the relationship between pain during EMG and the degree of anxiety of patients are experiencing still contradictory ${ }^{24}$.
During the project design, the latency of tramadol was considered (30 $\mathrm{min})$ as sufficient to initiate EMG under the effects of the drug.

It is known that the maximum plasma concentration of the drug is reached within $2 \mathrm{~h}^{25}$, which leads us to think that EMG and evaluation of pain intensity could be done with a sufficient plasma concentration but suboptimal to achieve the maximum benefits of an analgesic drug.

Although the calculation of the initial sample was 30 patients per group, it was decided to conclude the inclusion of patients at $n=57$ because the tendency observed by previous statistical analyses showed no difference between treatment groups, and by this moment, it had a sufficient potential level $(P=85.3 \%)$ established to priority.

As for the limitations of this research, we can consider the type of sampling. Purposeful sampling of patients who would come to the Rehabilitation Department of Hospital General de México to ask for an EMG could induce a non-differential selection bias study of a specific sample. This specific selection sample improves the internal validity of the research but reduces external validity. Therefore, the results can only be generalized to the type of population of Hospital General de México, and specifically, only those that require an EMG in the lower back and/or pelvic extremities.

To date, pain assessment remains complex because it is a subjective phenomenon ${ }^{22}$. The application of simple scales such as VAS is useful tools for converting the subjectivity of the phenomenon to an objective variable. One-dimensional scales to measure pain intensity such as VAS, the numerical scale, face recognition, or verbal descriptors are considered ordinal variables. However, VAS is a $10 \mathrm{~cm}$ "closed" scale that contains universal values the examinee could present, which is why it has been used conventionally as a continuous numeric vari$a_{b l e}{ }^{26}$. Consequently, the statistical analysis of the results of VAS could be performed with non-parametric statistics if it is considered as an ordinal variable, or with parametric statistics, as in the case of our study.

At present, an alternative to a more objective measurement of pain perception is brain imaging by positron emission tomography so that the subjective phenomenon of pain is expressed in localizing brain activation during pain stimulation. However, the design of a paradigm similar to that in EMG and the cost of such studies are limitations to the use of this technology ${ }^{22}$. The evaluation of other analgesic drugs or their combination (e.g., NSAIDs + opioids) could provide better results than those obtained in our study due to the acute inflammatory process generated by nociceptive stimulation by the 
introduction of the needle in the muscle fiber, with the release of algogenic substances (such as serotonin, bradykinin, histamine, and substance P) that are not modified by the tramadol mechanism of action ${ }^{25}$. In our study, the choice of tramadol as a useful analgesic was due to a non-theoretical pragmatic model of pain caused by the EMG and the mechanism of action of the drug.

So far, researchers have made several attempts to reduce the discomfort generated by this procedure but have been unable to obtain satisfactory results that could be generalized in clinical practice ${ }^{5,9,10,27}$. Today, EMG continues to be a necessary tool for the diagnosis of patients with neuromuscular diseases and cannot be replaced by other less painful or painless studies. However, doctors cannot remain indifferent to the pain of the patient; it is necessary for researchers to look for alternatives to avoid and to have a greater understanding of the complex experience of pain.

\section{Conflicts of interest}

The authors declare no conflicts of interest.

\section{Funding}

The authors declare that this research did not receive any specific grant from funding agencies in the public, commercial, or non-profit sectors.

\section{Ethical disclosures}

Protection of human and animal subjects. The authors declare that the procedures followed were in accordance with the regulations of the relevant clinical research ethics committee and with those of the Code of Ethics of the World Medical Association (Declaration of Helsinki).

Confidentiality of data. The authors declare that they have followed the protocols of their work center on the publication of patient data.

Right to privacy and informed consent. The authors have obtained the written informed consent of the patients or subjects mentioned in the article. The corresponding author is in possession of this document.

\section{References}

1. Preston D, Shapiro B. Electromyography with needle electrode. In: Katirji B, editor. Neurologic Clinics: clinical Electromyography. Cleveland: Mc Graw Hill-Interamericana; 2002. p. 355-93.

2. Rubin DI. Needle electromyography: basic concepts and patterns of abnormalities. Neurol Clin. 2012;30:429-56.

3. Dumitru D. The Needle electromyography. In: Dumitru D, editor. Electrodiagnostic Medicine. $9^{\text {th }}$ ed. Philadelphia, PA: Hanley and Belfus Inc.; 1994. p. 211-324.

4. Ortiz-Corredor F. Clinical examination and electromyographic abnormalities in patients with lower back pain. Rev Neurol. 2003;37:106-11.

5. Richardson JK, Evans JE, Warner JH. Informational effect on the perception of pain during electromyography. Arch Phys Med Rehabil. 1994;75:671-2.

6. Wee AS, Boyne RL, Albernathy SD, Nick TG. Pain perception to nerve conduction and needle electromyographic procedures. J Miss State Med Assoc. 2004; 45:327-30.

7. Balbierz JM, Petajan JH, Alder SC, Vlach SA. Differences in pain perception in women using concentric and monopolar needles. Arch Phys Med Rehabil. 2012;87:1403-6.

8. Kim S, Kim W, Kim H, et al. Abnormal spontaneous activities on needle electromyography and their relation with pain behavior and nerve fiber pathology in a rat model of lumbar disc herniation. Spine. 2011;36:1562-7.

9. Murphy B, Dawson N, Irwin RJ. Intramuscular sensation in conscious human subjects: a qualitative and quantitative study utilizing signal detection theory methodology. Somatosen Mot Res. 2002;19:181-90.

10. Nijs J, Daenen L, Cras P, Struyf F, Roussel N, Oostendorp RA. Nociception affects motor output. A review on sensory-motor interaction with focus on clinical implications. C J Pain. 2012;28:175-81.

11. Banerjee M, Bhaumik DJ, Ghosh AK. A comparative study of oral tramadol and ibuprofen in postoperative pain in operations of lower abdomen. $\mathrm{J}$ Indian Med Assoc. 2011;109:619-22.

12. Dhillon S. Tramadol/paracetamol fixed-dose combination: a review of its use in the management of moderate to severe pain. Clin Drug Invest. 2010;30:711-38.

13. Gobel H, Stadler T. Treatment of pain due to postherpetic neuralgia with tramadol. Results of an open parallel pilot study versus clomipramine with or without levomepromazine. Clin Drug Invest. 1995;10:208-14.

14. Jick H, Derby L, Vasilakis C, Fife D. The risk of seizures associated with tramadol. Pharmacotherapy. 1998;18:607-11.

15. Kahn LH, Alderfer RJ, Graham DJ. Seizures reported with tramadol. JAMA. 1997;278:1661-8.

16. Lehman KA. Tramadol for the management of acute pain. Drugs. 1994; 47 Suppl 1:19-32.

17. Schulz KF, Altman DG, Moher ID, et al. Group. CONSORT 2010 statement: updated guidelines for reporting parallel group randomized trials. BMJ. 2010;340:c332.

18. Delagi E. The limbs and trunk. In: Perotto A, Delagi E, lazetti J, editors. Anatomical Guide for the Electromyographer. 3rd ed. Springfield: Thomas C Publisher; 2006. p. 354-421.

19. El-Salem K, Shakhatreh M, Prospective double-blind crossover trial of ibuprofen in reducing EMG pain. Muscle Nerve. 2008;38:1016-20.

20. Smith MJ, Tong HC. Manual acupuncture for analgesia during electromyography: a pilot study. Arch Phys Med Rehab. 2005;86:1741-4.

21. Annaswamy TM, Morchower AH. Effect of lidocaine iontophoresis on pain during needle electromyography. Am J Phys Med Rehabil. 2011;90:961-8.

22. Wylde E, Plamer S, Learmonth I, Dieppe P. Test-retest reliability of quantitative sensory testing in knee osteoarthritis and healthy participants. Osteoarth Cartilage 2011;19:655-8.

23. Walker WC, Keyser-Marcus LA, Johns JS, Seel RT. Relation of electromyography-induced pain to type of recording electrodes. Muscle Nerve. 2001;24:417-20.

24. Slack D, Nelson L, Patterson D, Burns S, Hakimi K, Robinson L. The feasibility of hypnotic analgesia in ameliorating pain and anxiety among adults undergoing needle electromyography. Am J Phys Med Rehabil. 2009;88:21-9.

25. Power I. An update of analgesics. Br J Anaesth. 2011;107:19-24.

26. Breivick $\mathrm{H}$, Borchgrevinck PC, Allen SM, et al. Assessment of pain. Br J Anaesth. 2008;101:17-24.

27. Pease WS, Bowyer BL. Motor unit analysis. Comparison between concentric and monopolar electrodes. Am J Phys Med Rehabil. 1988;67:2-6. 